\title{
Das Verhalten des kindlichen Brustkastens bei der Geburt.
}

Von

\section{Hermann.}

Bernstein hat vor einigen Jahren ${ }^{1}$ ) die Angabe gemacht, dass der Thorax des Neugeborenen nach der ersten Inspiration (welche er an der Leiche dureh Einblasen von Luft mit einem Blasbalg ersetzte) eine bleibende, mit dem Fühlhebel nachweisbare Erweiterung erfahre. Diese Erweiterung erkläre das plötzliche Auftreten der Aspiration des Thorax, welche vor der ersten Athmung nicht vorhanden war, nach der Lufteinblasung aber (durch die Spannung der Lungenluft nach Oeffnung des Thorax, Donders) von Beruste in nachgewiesen wurde. Zur Erklärung dieser plötzlichen bleibenden Thoraxerweiterung durch die erste Atbmung hat Bernstein zwei Möglichkeiten aufgestellt: erstens einen sperrzahnartigen Mechanismus der Rippengelenke, zweitens eine Ueberschreitung der Elasticitätsgrenzen des Thorax, welche zu einer Ueberdehnung führe.

Ich habe dann darauf aufmerksam gemacht ${ }^{2}$ ), dass von Bernstein ein Moment gảnzlich übersehen worden ist, welches eine bleibende Erweiterung des Thorax durch die erste Athmung ganz unzweifelhaft herbeifübrt, nämlich die bleibende Erweiterung der Lunge selbst. Die Lunge ist vor der ersten Athmung atelectatisch und sinkt in Wasser unter, nach der ersten Athmung aber bleibend lufthaltig und schwimmt anf Wasser. Ich zeigte ferner mit $O$. Keller, dass wenn etwa schon vor der ersten Athmung eine Aspiration des Thorax existirte, diese die Lunge nicht zu entfalten im Stande wäre, weil, wie unsre Versuche ergaben, eine

1) Dies Archiv, Bd. XVII, p. 617.

2) Dies Archiv, Bd. XX, p. 365. 
atelectatische Lunge ${ }^{1}$ ) wegen der Adhäsion und Verklebung der Bronchialwände ungleich grössere Kräfte zu ihrer Entfaltung beansprucht, als eine schon lufthaltige.

Ferner habe ich, da mir aus Bernstein's Versuchen hervorzugehen schien, dass der Thorax des Neugebornen nach der ersten Respiration den sogenannten aspiratorischen Zustand besitzt, dargelegt, dass wenn dies so ist, kein Grund existirt, anzunehmen, dass nicht diesẹr Zustand schon vor der ersten Athmung bestehe. Denn das Nichteindringen von Luft in die Lunge des Todtgebornen ist kein Gegengrund, da dasselbe durch die Adhäsion und Verklebung der Bronchialwände verhindert werden wïrde.

Hiergegen hat Bernstein vor Kurzem eine Reihe von Bemerkungen und Einwänden gemacht ${ }^{2}$ ). Was wir durch sorgfältige Versuche festgestellt haben, der Widerstand, den die Adhäsion und Verklebung der Bronchialwände dem Eintritt und noch mehr dem vollständigen Wiederanstritt der Luft, selbst bei hohem Druck, entgegengesetzt, ist für Bernstein etwas so Selbstverständliches, dass es unsers Nachweises kaum bedurft hätte (S. 230), und gebört nach ihm überhaupt gar nicht zur Sache (S. 238). Jener so selbstverständliche Umstand aber, der merkwürdigerweise von Bernstein mit keiner Silbe erwähnt wurde, ehe wir ihn hervorhoben, jetzt aber in seinen eigenen Argumentationen eine nicht unbedeutende Rolle spielt (z. B. S. 241), wäre mir, wenn ich nicht sehr irre, von Bernstein als ganz unbewiesene Behauptung bezeichnet worden, wenn ich keine Versuche dafür beigebracht hätte; schon deshalb警halte ich diese Versuche für nicht vergeblich, ganz abgesehen davon, dass ich die Sache nicht für so selbstverständlich ansehe, und die Versuche doch auch für die Grösse jenes Verklebungswiderstandes einen Anbalt geliefert haben. Wieso die unzweifelhafte und nachgewiesene, bleibende Volumzunahme der Lunge durch die erste Athmung nicht in Betracht kommen soll, wenn man nach Momenten sucht, welche die bleibende Volumzu-

1) Warum die von uns angegebene Methode der mechanischen Atelectase, die sich an der gleichen Lunge mehrmals wiederholen lässt, schlechter sein soll, als das von Brücke erwähnte, in seinen Bedingungen noch unklare und nicht wiederholbare Verfahren der Herstellung von Pneumothorax am lebenden Thiere, ist mir nicht ganz klar (vgl. Bernstein, dies Archiv, Bd. XXVIII, p. 239).

2) Dies Archiv, Bd. XXVIII, p. 229. 
nahme des Thorax mit Lunge durch die erste Athmung zu erklären geeignet sind, ist mir völlig unverständlich; dies Moment ist doch wohl solider, als so fernliegende und hypothetische Dinge wie sperrzahnartige Rippengelenke oder gewaltsame Ueberdehnung.

Weiter behauptet Bernstein, das ron mir hervorgehobene Moment, welches trotz bestehender Aspiration die Entfaltung der Lunge zu verhindern geeignet ist, passe zwar fur den geborenen, aber nicht für den in Fruchtwasser befindlichen Fötús; denn wenn auch die Verklebung für Luft einen Widerstand biete, so sei dies nicht der Fall für Fruchtwasser. „Wenn Hermann und Keller versucht hätten, ihre atelectatischen Lungen mit Wasser statt mit Luft aufzublasen, so würden sie ohne Zweifel gefunden haben, dass hierzu nur ein Minimum von Druck erforderlich gewesen wäre." Hier muss ich aber sagen: Probiren geht über Studiren; warum hat Bernstein diesen Versuch nicht angestellt, dessen Resultat er so kühn voraussagt? Mit vagen Vergleichungen wie Gummischleim und Bronchialsecret ist hier, wo auf die Grösse des Adhäsions- und Verklebungswiderstandes Alles ankommt, und wo Experimente entscheiden können, Nichts anzufangen. So ist also ohne directen Versuch keineswegs zuzugeben, dass, wenn der fötale Thorax Aspiration besässe, er im Uterus Fruchtwasser ansaugen, also der Fötus ertrinken mïisste. Der Ausdruck „Ertrinken" scheint mir übrigens hier, wo von Erstickung keine Rede ist, nicht recht am Platze, wie schon B. S. Schultze ${ }^{1}$ ) mit Recht hervorgehoben hat.

Meine Bemerkung, dass die elastischen Kräfte des (aspirirend vorausgesetzten) Thorax, ,nachdem der intrauterine Druck durch die Geburt beseitigt ist", zur Entfaltung der Lunge nicht genügen, veranlasst Bernstein, bei mir die irrige Ansicht zu vermuthen, dass die Compression durch den Fruchtwasserdruck die Entfaltung verhindere, obwohl doch dieser Druck auch an den Zugängen zur Lunge wirkt. Bernstein hätte nicht nöthig gehabt, mir eine physikalisch unrichtige Meinung zuzusehreiben; vielmehr habe ich die Wirkung des Uterusdruckes so verstanden, dass die durch ihn bedingte Lage des Fötus mit uiber der Brust gekreuzten Armen und gekrümmter Wirbelsäule eine extrem exspiratorische Thoraxstellung bedingt, welche mit der Geburt durch Wegfall des Uterus-

1) Der Scheintod Neugeborener. Jena 1871. S. 131. 
druckes aufhört. Ich wundre mich um so mehr, dass Bernstein anf diese Deutung meiner Worte nicht gekommen ist, als er selber in der neuen Arbeit (S. 240, 241) eine ganz ähnliche Ansicht ausspricht.

Das eigentliche Novum, welches die letzte Mittheilung Bernste in's enthält, ist aber der Nachweis, dass vor der ersten Athmung keine Aspiration des Thorax bestehe. In der ersten Mittheilung hatte Bernstein dies zwar behauptet, aber ohne experimentellen Beweis, lediglich auf Grund von Erwägungen, bei denen die Adhäsion der Bronchialwände und die Lage des Fötus im Uterus unberücksichtigt geblieben war, so dass die Behauptung durchaus zweifelhaft erscheinen musste. Jetzt führt er fiur dieselbe Versuche an; er setzt nämlich die Pleurahöhle des Todtgebornen in Communication mit einem mit Flüssigkeit gefüllten Manometer, und fincet, dass an diesem keine Saugkraft zu erkennen ist. Obwohl mir bei diesem Versuch das Moment der Adhäsion der Pleurawände nicht gentigend eliminirt scheint (bei ausgiebiger Oeffnung des Thorax Todtgeborner entfernen sich nämlich in der That die Brusteingeweide etwas von der Thoraxwand, s. unten), will ich doch den von Bernstein gezogenen Schluss, dass der fötale Thorax keine merkliche Aspiration zeigt, acceptiren, darum nämlich, weil ich, wie sogleich zu berichten ist, gefunden habe, dass selbst nach der ersten Athmung keine Aspiration vorhanden ist, wodurch sich die ganze Frage fundamental umgestaltet. Aus gleichem Grunde habe ich es auch nicht für nöthig gehalten, den von Bernstein unterlassenen Versuch anzustellen, ob wirklich eine atelectatische Lunge sich leichter mit Fruchtwasser als mit Luft füllen lässt, oder gar mit ersterem unter einem Minimum von Druck, wie Bernstein behauptet.

In meiner ersten Arbeit habe ich den Standpunct innegehalten, dass kein Grund vorhanden ist, eine plötzliche Veränderung der Thoraxgrösse in Folge der ersten Athmung anzunehmen, und da ich Bernstein's Angabe für richtig hielt, dass nach der ersten Athmung der aspiratorisehe Zustand entwickelt sei, folgerichtig: vermuthet, dass er schon vorher vorhanden sein möchte. Da nunmehr letzteres direct ausgeschlossen scheint, muss der angeführte Standpunct umgekehrt zu der Frage führen, ob denn wirklich nach der ersten Athmung der aspiratorische Zustand vorhanden ist, wie ich auf Bernstein's Angabe hin bisher geglaubt hatte. 
Daher habe ich nun tiber diesen Punct directe Versuche angestellt, und bin damit zugleich einem Wunsche Bernstein's entgegengekommen, welcher solche Versuche - wie mir seheint mit Unrecht, da ich mich im Thatsächlichen eben auf ihn verlassen hatte, - in meiner ersten Arbeit vermisst hat (S. 230). Ich bin sehr froh, solche Versuche angestellt za haben; die ganze von Bernstein hervorgehobene Schwierigkeit der Entstehung der Aspiration durch die erste Athmung fällt nämlich durch sie in einfachster Weise dahin, weil Bernstein's Hauptergebuiss, dass nach der ersten Athmung Aspiration vorhanden ist, völlig unrichtig ist.

Anstatt aber wie Bernstein die Lungen todtgeborner Kinder mit dem Blasbalg aufzublasen, zog ich den weit natïrlicheren Weg vor, die Leichen soleher Neugebornen auf Aspiration des Thorax zu untersuchen, welche erst einige Zeit nach der Geburt gestorben waren, und unzweifelhaft und kräftig geathmet hatten. Von diesen Leichen, welche mir durch die Güte meines Freundes Frankenhäuser zur Verfügung standen, war die jüngste in der ersten Stunde, die älteste erst 4 Tage nach der Geburt gestorben. In die Trachea wurde eine metallene, mit Riefen versehene Canüle gut eingebunden und dureh ein Gabehrohr mit einem Wasser- und einem Quecksilbermanometer luftdicht verbunden. Ein mittels eines T-Rohres seitlich communicirender Kautschnkschlauch gestattete einerseits den Druck in den Schläuchen jederzeit auf Null (Luftdruck) zu bringen, andrerseits später aus einem Gasometer Luft einzublasen. In diesen Schlauch und in den an die Trachealcanüle angefügten Hauptschlauch war ein gut schliessender Glashahn eingeschaltet. Ein Hahn ist viel besser als eine Klemme, weil letztere beim Schliessen und Oeffnen Veränderungen am Wassermanometer hervorbringt. Alle Schläuche waren so kurz wie möglich.

Nachdem im Schlanchsystem Luftdruck hergestellt und der seitliche Hahn geschlossen ist, wird anch der Haupthahn geschlossen und nun der Thorax auf das Sorgfältigste weit geöffnet. Jetzt wird der Haupthahn geöffnet und es zeigt sich nicht das geringste Ansteigen des Manometers. Darauf wird der Seitenhahn geöffnet und die Lunge fällt nicht im mindesten zusammen, sondern erfüllt nach wie vor den Thorax ganz. Der sehr geringe Raum zwischen Brustwand und Lunge, der schon bei völlig abgeschlossener Tra- 
chea vorhanden ist, erklärt sich einfach aus dem Einfluss der Schwere, welchem die weichen Eingeweide stärker unterliegen als die starre Thoraxwand. Legt man den Oberkörper etwas tiefer, so dass die Baucheingeweide stärker gegen das Zwerchfell drängen, so verschwindet jener kleine Raum, oder die Brusteingeweide quellen sogar hervor. Am Schlusse jedes Versuches wurde die Lunge mittels des Gasometers vollständig aufgeblasen, um ihre Unversehrtheit zu constatiren; schon geringer Druck genügt zur vollständigsten Entfaltung.

Dieser Versuch, der jedesmal dasselbe Resultat gab, zeigt, dass auch nach der ersten Athmung der Thorax des Neugeborenen keine Aspiration in der Leichenstellung besitzt; die Lunge, welche den Thorax ganz erfüllt, sinkt auch nach Eröffnung des Thorax nicht zusammen. Oder mit anderen Worten: In den ersten Lebenstagen existirtkeine sog. Residualluft, ausser derjenigen, welche überhaupt nicht ausgetrieben werden kann, und welche das Schwimmen der Lunge auf Wasser verursacht.

Durch welchen Umstand Bernstein seinem Irrthum verfallen ist, lässt sich leicht vermuthen. Wahrscheinlich dadurch, dass er das Aufblasen des fötalen Thorax mit dem Blasbalg für mechanisch gleichwerthig hielt mit der natürlichen Inspiration. Wenn er einen hohen Druck angewendet hat, so ist es leicht begreiflich, dass die Thoraxwände überdehnt und dadurch der Thorax bleibend erweitert wurde. Um diese Vermuthung zu prüfen, habe ich an einer Anzahl Leichen todtgeborner Kinder und an sehr vielen aus dem Schlachthause bezogenen Schafföten (dieselben wurden stets im unversehrten Uterus auf das Laboratorium gebracht und waren grösstentheils ausgetragen) künstliche Lufteinblasungen vorgenommen, und dann den Donders'schen Versuch angestellt. Die Aufblasung wurde mit einem Gasometer, unter Controlle eines an die Leitung seitlich angefügten Quecksilbermanometers vorgenommen. Oeffnet man den lufteinleitenden Hahn äusserst vorsichtig, bis man an der Leiche deutliches Anschwellen erkennt (am deutlichsten am Epigastrium), lässt nun die eingetriebene Luft wieder entweichen, und stelit dann den Versuch wie oben angegeben an, so findet man meist, obwohl die Lungen sich sehön lufthaltig erweisen, nicht den mindesten Donders'schen Druck, und kein Collabiren nach Freigabe des Ausweges. Zur Eintreibung von Luft in den uneröfneten fötalen Thorax genügte meist ein Druck von $40-50 \mathrm{~mm}$ 
Hg. Wurde dagegen absichtlich ein höherer Druck angewandt, als zum Lufteintritt erforderlich war, sei es mit Blasbälgen, sei es durch Gasometerdruck, so ergab sich jedesmal ein Donders'scher Druck von 20-30 oder mehr mm Wasser. Es unterliegt also nicht dem mindesten Zweifel, dass Bernstein, welcher an menschlichen Neugebornen Donders'sche Driicke von 6-7 mm Hg, also 81-95 mm Wasser, beobachtet hat, die Luft unter ganz enormem Druck eingeblasen hat.

Ein Blick auf die Literatur der forensischen Leichenschau bei Neugeborenen zeigt übrigens, dass die erfahrensten Gerichtsärzte bisher die von Bernstein behauptete Thoraxvergrösserung durch den Umstand, dass der Neugeborne geathmet hat, nicht bemerkt haben. Casper z. B. fübrt eine Tabelle von 238 reifen Neugebornen (158 lebend and 80 todt geboren) an, aus welcher hervorgeht, dass die Thoraxdimensionen im Mittel beim.Lebendgebornen nicht grösser sind als beim Todtgebornen ${ }^{1}$ ). Das einzige einigermassen brauchbare Criterum, welches die Thoraxgrösse betrifft, ist nach ihm der grössere Tiefstand des Zwerchfells bei Lebendgebornen ${ }^{2}$ ) und in der That gesehieht wohl die geringe bleibende Vergrösserung des Thoraxvolums durch die erste Athmung, welche von der gefangenen Minimalluft herrührt (s. unten), wesentlich auf Kosten des Abdomens, wo viel weniger Widerstand zu überwinden ist, als durch Verstellung des Rippenkorbes, wie sie Bernstein an seinen künstlich aufgeblasenen Föten fand. Bei Beschreibung des Anblicks, den der geöffnete Thorax des Lebend- und des Todtgebornen gewährt, sagt Casper ${ }^{2}$ ): „Es ist allgemein bekannt, dass fơtale Lungen, wenn man die vordere Brustwand entfernt hat, so liegend gefunden werden, dass sie die Brusthöhle nicht ausfüllen, und dass namentlich die linke das Herz auch nicht theilweise deckt, während die Lungen nach der Athmung den Thorax um so mehr ausfüllen, je vollständiger die Respiration eingeleitet gewesen ist u. s. w." Wäre Bernstein's Angabe richtig, so mïsste bei Oeffnung des Thorax vor Allem auffallen, - und Casper hätte nicht unterlassen dies anzuführen,

1) Casper, Practisches Handbuch der gerichtlichen Medicin. 4. Aufl. Berlin 1864, II. p. 759 ff.

2) a. a. 0. p. 764 .

3) a. a. 0. p. 766 . 
- dass beim Lebendgebornen die Lunge zurticksinkt und den Thorax nicht ausfüllt, während sie beim Todtgebornen nicht zurücksinkt und den Thorax ausfüllt. Nichts davon ist zu bemerken; das Klaffen zwischen Brustwand und Brusteingeweiden ist bei Todt- und Lebendgebornen gleich gering (durch das Zurückweichen des Zwercbfells bedingt, s. oben), so dass als Unterschied nur die verschiedene Grösse der Lungen und ihr Verhältniss zum Herzen auffällt; letzteres bezeichnet Casper durch den sehr missdeutungsfähigen Ausdruck, dass beim Todtgebornen die Lunge den Thorax nicht ausfüllt; nach Bernstein müsste im Gegentheil gesagt sein, dass beim Lebendgebornen die Lunge den Thorax nicht ausfüllt.

Die natürliche Inspiration des Neugebornen erweitert also, wie aus den zuletzt mitgetheilten Versuchen hervorgeht, den Thorax durchaus nur innerhalb seiner Elasticitätsgrenzen, so dass er nach dem Aufhören der Inspirationskraft wieder zum alten Volumen zurïckkehren würde, wenn nicht ein Quantum Luft in der einmal entfalteten Lunge durch die Adhäsion der Bronchialwände dauernd gefangen bliebe, so dass die Lunge nunmehr auf Wasser schwimmt. Die bleibende Erweiterung, welche der Thorax in Folge der ersten Inspiration erfährt, ist also sehr gering, und entspricht nur der eben angeführten Volumzunahme der Lunge durch die gefangene Luft.

Um die physiologische Bedeutung dieser Thatsache zu würdigen, wird es zweckmässig sein, einige neue Benennungen einzuführen. Für den Zustand der Lunge, wie er bei jeder Leiche nach Eröffnung des Thorax sich herstellt, fehlt es an einer allgemein recipirten Bezeichnung; manche Autoren nennen die so collabirte Lunge atelectatisch, doch sollte dieser Ausdruck nur für die wirklich Iuftleere, in Wasser untersinkende, z. B. die fơtale Lunge angewandt werden. Ferner bezeichnet Residualluft den ganzen im Zustande tiefster Exspiration in der Lunge vorhandenen Luftvorrath, und es fehlt an einer Unterscheidung der beiden Bestandtheile dieses Vorrathes, nämlich des durch Collabiren bei Oeffnung des Thorax austreibbaren, und des überhaupt nicht austreibbaren.

Ich erlaube mir nun den Vorschlag, die Bezeichnung „atelectatisch" oder besser "anectatiseh" ausschliesslich für den Zustand der völlig luftleeren, in Wasser untersinkenden Lunge beizubehalten, die lufthaltige, collabirte, auf Wasser schwimmende Lunge (wie bei Pneumothorax) aber als "protectatisch" zu be- 
zeichnen, weil der betreffende Ausdehnungsbetrag durch den ersten Lufteintritt gleichsam bedingt und für ihn characteristisch ist. Den Luftgehalt der protectatischen Lunge möchte ich die ,Minimall $\mathrm{uft}^{\text {" }}$ nennen. Die Luft ferner, welche nach stärkster Exspiration nur durch Pneumothorax entleert werden kann, nenne ich „Collapsluft", weil sie durch Collabiren der von den Thoraxfesseln befreiten Lunge entleert wird. Der Ausdruck ,Residualluf $\mathrm{t}^{\text {" }}$ kann für die nach starker Exspiration in der Lunge zurïckbleibende Luft beibehalten werden, so dass Residualluft $=$ Collapslaft + Minimalluft. Die Benennungen haben den Vortheil, einerseits Missverständnisse zu beseitigen, andrerseits die hergebrachten Ausdrticke beizubehalten, mit der Fixirung für den Fall, für den sie ursprünglich aufgestellt waren. Dem Neugebornen fehlt hiernach die Collapsluft, oder es ist für ihn Residualluft = Minimalluft. Folgende Schemata verdentlichen das Gesagte ${ }^{1}$ ).

Für den Erwachsenen:

Tiefste Inspiration

Gewöhnliche Inspiration

Gewöhnliche Exspiration

Tiefste Exspiration .

Protectase . . .

$\left.\cdot \frac{\text { Complementärl }}{\frac{\text { Athmungsluft }}{\text { Reserveluft }}} \mid \begin{array}{l}\text { Collapsluft } \\ \text { Minimalluft }\end{array}\right\}$ Residuallupacit.

Anectase .

Für den Neugebornen:

Tiefste Inspiration

Complementärl.

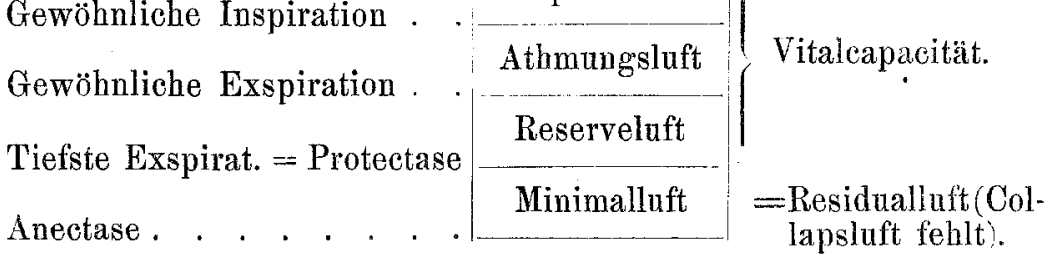

1) Auf das Verhältniss der polumgrössen ist keine Rücksicht genommen. 
Bei den oben angefühiten Manometerversuchen zeigt die Lange nach Oeffnung des Brustkastens nicht allein keinen positiven, sondern auch keinen negativen Druck am endständig eingebundenen Manometer, d. h. sie war im Brustkasten weder über ihr natürliches Volum gedehnt, noch unter dasselbe comprimirt (was denkbar wäre, da sie lufthaltig ist); es herrscht demnach im Thorax des Neugebornen, welcher geathmet hat, in der Leichenstellung; soweit nachweisbar, Druckgleichgewicht. Trotzdem ist dasselbe vielleicht kein absolutes, wie folgende Betrachtung lehrt. Man darf wohl erwarten, dass im Fötalzustande das Gleichgewicht absolut ist, d. h. dass Lunge und Thorax ohne allen gegenseitigen Zwang sich einander entsprechend ausbilden. Das geringfügige Zuruickweichen der Brnsteingeweide, welches man nach Oeffnung des Thorax eines Todtgebornen, wie oben erwähnt, in der Regel bemerkt, ist kein Gegengrund, da die Schwere allein zu dessen Erklärung hinreicht. Wenn das Gleichgewicht aber vor der ersten Athmung vollkommen war, so darf erwartet werden, dass nach derselben die bleibende Vergrösserung der Lunge durch die gefangene Minimaliuft einen geringen positiven Druck im Thorax macht. Der Nachweis desselben dürfte aber kaum möglich sein. Die Lungenluft wïrde durch ihn nach Oeffnung des Thorax negativen Druck zeigen; diesen kann aber ein Trachealmanometer selbstverständlich nicht anzeigen, da ja die Adhäsion der Bronchialwände so gut wie das Ausströmen der Minimalluft (s. meine frühere Arbeit mit 0 . Keller) auch das Einsaugen von Luft verhindern muss; das Manometer miisste also direct mit den Alveolen communiciren. Der Bernstein'sche Pleuraversuch kann ebenfalls positiven Thoraxdruck nicht anzeigen, da es an pleuraler Flüssigkeit fehlt, die in die Canïle eindringen könnte. Allenfalls könnte eine Prüfung an den Venen in Frage kommen, doch müssten vor Oeffnung des Thorax alle aus ihm austretenden Gefässe, bis auf die Versuchsvene, verseblossen sein, was grosse Schwierigkeiten hätte. Uebrigens ist der zu vermuthende positive Druck wahrscheinlich sehr klein, wegen des geringen Betrages der Minimalluft. Die genauere Feststellung dieses Betrages, und zwar in verschiedenen Lebensaltern, ist übrigens eine noch ungelöste Aufgabe; ich habe die Absicht, in dieser Richtung Untersuchungen zu veranlassen.

Sehen wir ab von etwaigen durch die Todtenstarre bedingten Complicationen, so können wir nunmehr behaupten, dass der Thorax 
des Neugeborenen das beständige Bestreben hat, die Lungenluft fast vollständig (nämlich bis auf die Minimalluft) zu entleeren. Auch obne jede active Exspiration muss also die Ventilation der Lunge des Neugeborenen eine viel lebhaftere sein, als beim Erwachsenen. Während letzterer selbst bei dem kräftigsten Athmen mit activer Exspiration immer nur eine partiale Lufterneuerung in der Lunge hat, besitzt der Neugeborene auch bei bloss passivem Exspiriren eine fast integrale Lufterneuerung. Hierin liegt anscheinend eine grosse Zweckmässigkeit, da mit möglichst wenig Muskelarbeit möglichst energische Ventilation erreicht wird. Man wird hierdurch zu der Frage angeregt, welchen Vortheil ibberhaupt die beträchtliche Residualluft des Erwachsenen mit sich bringt. Soviel ich sehen kaun, lassen sich die Wirkungen der beim Erwachsenen bestehenden Einrichtung in folgende Puncte zusammenfassen: 1. Beständige Aspiration auf die Venen, auch im exspiratorischen Zustande (ausser wenn dieser mit vorgängigem Stimmritzenschluss verbunden ist); 2. Gleichmässigere, von den Athmungsphasen unabhängigere Zusammensetzung der Alveolenluft; 3. Vorhandensein eines gewissen Luftvorrathes in der Lunge auch bei plötzlicher Lähmung der Athembewegungen. Diese Wirkungen scheinen zugleich Vortheile zu sein; wenn dieselben beim Neugebornen preisgegeben sind, zu Gunsten des durch die fast integrale Ventilation unzweifelhaft bedingten viel höheren Sauerstoffgehalts der Alveolenluft, so ist zu vermuthen, dass es auf diesen beim Nengeborenen in erster Linie ankommt; vermuthlich hängt dies mit der längst bekannten grossen Lebhaftigkeit des Gaswechsels in der ersten Lebenszeit zusammen.

Aus den vorstehenden Angaben ergiebt sich eine nicht unwichtige Aufgabe, welche wir, soweit sich Material bietet, zu erledigen suchen werden. Es ist nämlich durch Anstellung des Donders'schen Versuches an Leichen der verschiedensten, namentlich kindlichen Lebensalter zu verfolgen, zu welcher Zeit der definitive aspiratorische Zustand des Thorax sich ausbildet. Offenbar kann man sagen, dass der Brustkasten schneller wächst als die Lungen; die Differenz beider Grössen stellt jederzeit den Betrag der Collapsluft dar, von welchem wieder die Stärke der Aspiration des Thorax abhängt.

Schliesslich mögen einige beiläufig gemachte Beobachtungen kurz erwähnt werden. Die Luftröhre todtgeborner menschlicher 
Föten ist, wie schon Bernstein richtig angiebt, platt zusammengedrückt. Dagegen ist die Luftröhre von Sehafsföten mit ihrer Hinterwand in die Vorderwand eingefaltet, und bildet in toto einen runden Strang; das doppelwandige Band, das beim menschlichen Fötus platt liegt, ist also beim Schafsfötus noch einmal der Länge nach gleichsam eingerollt. Druck auf den Thorax gab bei den von mir untersuchten menschlichen und Schafsföten ausnahmslos beträchtliches Ansteigen eines mit der Luftröhre endständig verbundenen, möglichst engen Wassermanometers $(80-100 \mathrm{~mm})$, anch in Fïllen wo ganz sicher kein Fruchtwasser in die Lungen gedrungen war (vgl. dagegen die Angabe Bernstein's S. 235). Icb erkläre mir dies so, dass beim Einbinden einer Canïle in die Luftröhre diese bis weit hinunter sich entfaltet und lufthaltig wird. - Endlich muss ich eine auffällige Erfahrung erwähnen, die schon in der friheren Mittheilung angedeutet worden ist (Bd. XX. S. 367). Die zahlreichen von mir untersuchten thierischen Embryonallungen zeigten, wenn sie bei geöffnetem Thorax oder nach Herausnahme aufgeblasen wurden, ausnahmslos schon bei sehr niedrigem Druck emphysematischen Luftaustritt aus dem Bronchialsystem.

\section{Nachschrift.}

Im Begriff diese Arbeit zum Druck abzusenden, erhalte ich die Gelegenheit ein Kind zu untersuchen, welches 8 Tage nach der Geburt in Folge eines Coccygealtumors gestorben ist, und stets gut geathmet hat. Der D on ders 'sche Versuch ergab einen Lungendruck von $6 \mathrm{~mm}$ Wasser $=0,4 \mathrm{~mm} \mathrm{Hg}$ nach Oeffnung des Thorax. Nach Freigebung der Trachea sank die Lunge nicht nachweisbar zusammen, sondern fullte nach wie vor den ganzen Thorax aus. Also auch bei diesem 8tägigen Kinde (das älteste im Text erwähnte war 4tägig) war bis anf eine zweifelhafte Spur noch keine Aspiration des Brustkastens entwickelt. 